La Revue

des Droits

de l'Homme

\section{La Revue des droits de l'homme}

Revue du Centre de recherches et d'études sur les droits fondamentaux

Actualités Droits-Libertés | 2010

\title{
Choix du nom porté par chaque époux
}

INTERDICTION DE LA DISCRIMINATION ET DROIT AU RESPECT DE LA VIE FAMILIALE (Art. 8 et $14 \mathrm{CEDH})$

\section{Nicolas Hervieu}

\section{OpenEdition}

Journals

Édition électronique

URL : http://journals.openedition.org/revdh/3604

DOI : $10.4000 /$ revdh.3604

ISSN : 2264-119X

\section{Éditeur}

Centre de recherches et d'études sur les droits fondamentaux

\section{Référence électronique}

Nicolas Hervieu, "Choix du nom porté par chaque époux ", La Revue des droits de l'homme [En ligne], Actualités Droits-Libertés, mis en ligne le 09 novembre 2010, consulté le 20 avril 2019. URL : http:// journals.openedition.org/revdh/3604; DOI : 10.4000/revdh.3604

Ce document a été généré automatiquement le 20 avril 2019

Tous droits réservés 


\section{Choix du nom porté par chaque époux}

INTERDICTION DE LA DISCRIMINATION ET DROIT AU RESPECT DE LA VIE FAMILIALE (Art. 8 et $14 \mathrm{CEDH})$

\section{Nicolas Hervieu}

Dans la perspective de leur mariage en Suisse, un ressortissant hongrois et une ressortissante suisse n'ont pu obtenir l'autorisation de continuer chacun à porter leur nom respectif. Dans le but de contester cette décision, ils tentèrent, en application des règles de droit international privé suisse, de faire régir le nom du futur époux par le droit hongrois ce qui aurait permis à ce dernier de conserver son nom. Puisque cette question était un obstacle à leur mariage, les intéressés changèrent de stratégie. Ils se marièrent donc en 2004 et optèrent pour le nom de l'épouse comme «nom de famille » (« Rose »), sans toutefois demander dans le même temps que l'époux conserve son seul nom (celui-ci se nomma alors «Losonci Rose»). Ce n'est que postérieurement qu'ils sollicitèrent le remplacement de ce double nom par le seul nom initial de l'époux et ce, sans que celui de l'épouse ne soit modifié. Le tribunal administratif saisi ne fit pas droit à cette demande car il refusa le cumul de deux options : la première, qui consiste à renverser le principe selon lequel « d'après le droit suisse, le nom du mari devient automatiquement le nom de famille » et donc à demander que « le nom de la femme soit le nom de famille et que celui ou celle ayant dû changer de nom à la suite du mariage $p$ [uisse] choisir de faire précéder le nom de famille de son propre nom (choix du double nom)» (§ 11) ; la seconde, prévue par le droit international privé suisse, qui permet de faire régir le nom par le droit national, en l'occurrence hongrois. En d'autres termes, en optant pour la première option, l'intéressé aurait renoncé à pouvoir exercer la seconde. Cette solution fut confirmée par le Tribunal fédéral suisse.

2 L'affaire dont a été saisie la Cour européenne des droits de l'homme s'insère dans une série de solutions rendues par le passé sur le terrain de la discrimination entre hommes et femmes au sujet du choix du nom de famille après un mariage (v. Cour EDH, $4^{\mathrm{e}}$ Sect. 16 novembre 2004, Ünal Tekilit c. Turquie, Req. n²9865/96; en particulier concernant la 
législation suisse : v. Cour EDH, Ch. 22 février 1994, Burghartz c. Suisse, Req. nº 16213/90; sur le principe d'unité familiale dans le choix du nom des enfants, v. Cour EDH, $2^{\mathrm{e}}$ Sect. Dec. G.M.B. et K.M. c. Suisse, Req. $\mathrm{n}^{\circ}$ 36797/97). Prolongeant cette jurisprudence, la Cour condamne ici la Suisse pour discrimination dans la jouissance du droit au respect de la vie familiale (Art. 14 combiné à l'article 8). Pour ce faire, les juges européens admettent tout d'abord aisément la recevabilité de la requête (\$ 29), l'applicabilité de l'article 8 aux faits de l'espèce étant notamment établie au motif que si ce texte «ne contient pas de disposition explicite en matière de nom, [...] en tant que moyen d'identification personnelle et de rattachement à une famille, le nom d'une personne n'en concerne pas moins la vie privée et familiale de celle-ci " (\$ $26 ; \S 51$ : «le nom, en tant qu'élément d'individualisation principal d'une personne au sein de la société, appartient au noyau dur des considérations relatives au droit au respect de la vie privée et familiale »; v. Cour EDH, $2^{\mathrm{e}}$ Sect. 2 février 2010, Taşkin et autres c. Turquie, Req. $\mathrm{n}$ - 30206/04 et s. - ADL du 3 février 2010). Au fond, après un rappel des principes relatifs à l'interdiction de la discrimination, particulièrement stricte lorsque cette dernière est " fondée exclusivement sur le sexe » (\$37-41 - v. Cour EDH, $1^{\mathrm{e}}$ Sect. 7 octobre 2010, Konstantin Markin c. Russie, Req. ${ }^{\circ}$ 30078/06 - ADL du 8 octobre 2010), la juridiction strasbourgeoise commence par fixer l'objet exact de la différence de traitement litigieuse : dans le droit suisse, un homme étranger ne peut opter pour l'application du droit de son Etat d'origine afin de conserver son nom s'il a, au préalable, exercé une première option afin que le " nom de famille " soit celui de sa femme; à l'inverse, une femme étrangère mariée à un suisse peut, elle, exercer cette option en faveur du droit étranger car, comme le droit suisse pose le principe que le «nom de famille» est celui du mari, elle n'aura pas à eu besoin d'user de la première option (\$43-44). Une telle précision est importante car cet arrêt ne condamne pas en soi le principe d'attribution du nom du mari, à supposer toutefois qu'il puisse être renversé sur décision des membres du couple (V. Cour EDH, précité, Ünal Tekilit c. Turquie). La Cour souligne ainsi que si « cette différence de traitement a été causée par la règle selon laquelle le nom du mari devient le nom de famille (article $160 \mathrm{du}$ code civil) ou, plus précisément, par le choix des époux de renverser cette règle ", "de telles règles peuvent s'avérer nécessaires en pratique et ne sont pas nécessairement en contradiction avec la Convention ». Ce sont les conséquences de la mise en œuvre de ces règles qui sont ici visées en ce qu'elles ont "dans le cas d'espèce empêché le requérant de garder son nom après le mariage, contrairement à ce qui aurait été le cas si les requérants avaient été de sexe inverse 》 (\$ 49).

3 Afin d'examiner si cette différence de traitement poursuivait « une justification objective » et surtout si « un rapport raisonnable de proportionnalité entre les moyens employés et le but visé» a été préservé (\$46), la Cour rappelle qu' « un consensus se dessine au sein des Etats membres du Conseil de l'Europe quant au choix du nom de famille des époux sur un pied égalité et que, à l'échelle internationale, les développements au sein des Nations unies quant à l'égalité des sexes se dirigent dans ce domaine spécifique vers la reconnaissance du droit pour chaque conjoint de conserver l'usage de son nom de famille original ou de participer sur un pied d'égalité au choix d'un nouveau nom de famille » ( $\$ 47$ - v. la législation française à ce sujet. Sur la question du « double tiret » v. CE, $2^{\mathrm{e}}$ et $7^{\mathrm{e}} \mathrm{SSR}, 4$ déc. 2009, Lavergne, $\mathrm{n}^{\circ} 315818$ - ADL du 17 décembre 2009 (2)). Or, en n'admettant pas que le mari étranger puisse cumuler les deux options précitées - choix du nom de l'épouse comme «nom de famille » et choix du droit étranger pour conserver son nom - ( $\$ 48)$, il en résulte que «le régime en vigueur en Suisse engendre une discrimination entre les couples binationaux, selon que c'est l'homme ou la femme 
qui possède la nationalité suisse » (\$ 53), d’où le constat de violation de l'article 14 combiné à l'article 8.

4 Avant de prononcer cette condamnation à l'unanimité, la Cour a également tranché un point intéressant. Dans cette affaire, le Tribunal fédéral suisse avait certes reconnu, au terme d'un contrôle de constitutionnalité, que les « dispositions [litigieuses], prises dans leur ensemble, étaient contraires au principe de l'égalité de traitement entre les sexes ». Mais il n'avait pas souhaité en tirer de conséquences au motif qu'il «ne pouvait pas introduire des modifications du droit du nom qui avaient été refusées par le législateur » («le Parlement fédéral avait rejeté, le 22 juin 2001, une révision qui avait pour but de rendre le droit du nom conforme à la Constitution »- \$14). Les juges du Palais des droits de l'homme affirment en réponse et de façon lapidaire que « ceci ne change toutefois en rien la responsabilité internationale de la Suisse au titre de la Convention" ( $(50)$. Sans que cette position ne soit surprenante ni même inédite, le rappel de ce qu'un Etat ne peut écarter sa responsabilité en invoquant ses règles constitutionnelles peut être utile au regard de l'affaire relative à l'interdiction de construction de nouveaux minarets en Suisse. Sans se prononcer sur la recevabilité des requêtes déposées dans cette affaire ô combien brûlante (v. Cour EDH, $1^{\mathrm{e}}$ Sect. Association "Ligue des musulmans de Suisse" et autres c. Suisse, Req. n 66274/09 ; Hafid Ouardiri c. Suisse, Req. $\left.\mathrm{n}^{\circ} 65840 / 09\right)$, il est en tout cas certain que, contrairement à ce qui a été parfois avancé, l'origine référendaire de l'interdiction litigieuse et donc sa valeur constitutionnelle en Suisse ne sauraient être utilement invoquées par l'Etat défendeur comme moyen de défense à Strasbourg.

Losonci Rose et Rose c. Suisse (Cour EDH, $1^{\mathrm{e}}$ Sect. 9 novembre 2010, Req. $\mathrm{n}^{\circ}$ 664/06)

\section{AUTEUR}

NICOLAS HERVIEU

CREDOF - Université de Paris Ouest Nanterre la Défense 